\title{
An unusual diagnosis of a bicornuate unicollis uterus
}

\author{
Costas Panayotidis • Jean-Michel Foidart • \\ Michelle Nisolle
}

Received: 4 May 2007 / Accepted: 5 July 2007 / Published online: 15 August 2007

(C) Springer-Verlag 2007

\begin{abstract}
We present a case of an unusual diagnosis of a bicornuate unicollis uterus mimicking a uterus didelphys with blind hemivagina. Few cases of a bicornuate uterus have been reported with the primary symptom of a paracervical pyocolpos. The difficulties involved in the diagnosis and management of this particular congenital malformation are described in detail. The role of imaging techniques and adequate preoperative preparation is emphasised with a review of recent literature.
\end{abstract}

Keywords Müllerian malformation .

Bicornuate unicollis uterus $\cdot$ Pyocolpos .

Blind hemivagina $\cdot$ Laparoscopy

\section{Case study}

A 28-year-old nulliparous woman attended the accident and emergency department with severe low pelvic pain and unusual vaginal discharges. She had been diagnosed at birth with agenesis of the left radius and cubitus (radial club hand) and during her childhood with dextrocardia, hypothyroidism and left renal agenesis at the age of 11 . She had a significant past surgical history of orthopaedic and plastic

C. Panayotidis $(\bowtie)$

Minimal Access Surgery, Department of Obstetrics

and Gynaecology, University of Manchester,

Manchester, UK

e-mail: costapan@hotmail.com

J.-M. Foidart $\cdot$ M. Nisolle

Department of Gynaecology and Obstetrics, Centre Hospitalier

Regional de Citadelle, University of Liege,

Boulevard du 12me De Ligne 1,

4000 Liege, Belgium surgery for her left arm. She had normal menarche and previous normal gynaecological examinations. However, she started to suffer from worsening deep pelvic pain and unusual vaginal purulent bleedings the day before. She was haemodynamically stable and pregnancy was excluded. The speculum examination was very painful for the patient; however, the cervix was not visualised. The digital examination revealed a smooth wall mass deep inside the vagina in front of the cervix. She was admitted to the hospital and symptomatic treatment was given for analgesia with antibiotic cover for possible pelvic infection. Further imaging investigations were requested in order to determine the origin of this bulgy mass. The ultrasound scan discovered an enlarged bicornuate aspect uterus and two locations of liquid collection $3 \times 4 \mathrm{~cm}$, thought to be abscesses without relation to the adnexa. The first was located at the left proximal part of the cervix and the other was located on the high anterior and median vaginal part (as paravesical location). There was no other adnexal mass or free fluid in the pouch of Douglas. The computed tomography (CT) scan revealed a bicornuate uterus with two left paracervical abscesses of 3-4 cm. It was not clear if there was one or two cervixes because of the anatomical distortion caused by the paracervical abscess (Figs. 1 and 2). She had appropriate counselling about the need for a minimal access investigation with laparoscopy and hysteroscopy in order to diagnose exactly what type of malformation was present and to assess the management options with evacuation or aspiration of these two abscesses. Until this point it was thought that she had a didelphys uterus with a blind hemivagina. Under general anaesthesia the vaginal examination revealed a large vaginal protruded cystic mass of about 4-5 cm. Pus was excreted through a small fistula from the anterior part of the vagina but not from the main bulgy mass. Therefore, pyocolpos was 
Fig. 1 CT scan images: bicornuate uterus with left paracervical abscess
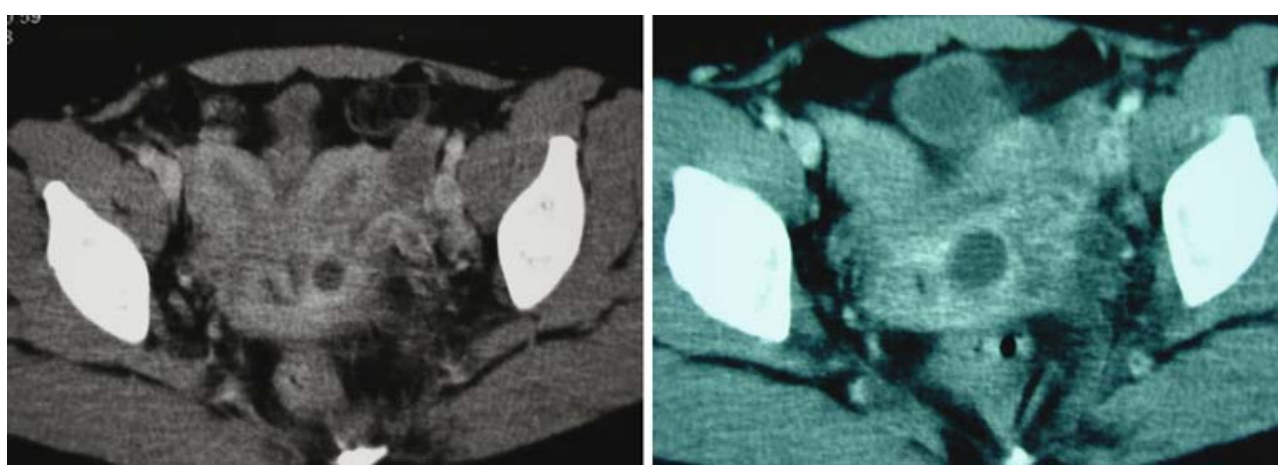

diagnosed but it was impossible to ascertain the anatomical relation with the uterus. The diagnostic laparoscopy revealed a bicornuate uterus with accentuated long horns equal in shape and volume for each side (Fig. 3). Both of the horns had a common union at the edge of the uterine body. The left ovary had a large luteal cyst and her right ovary was normal. Both fallopian tubes were normal and equal in length. A relatively thick appendix was noted. No signs of pelvic inflammatory disease (PID), endometriosis, adhesions or blood in the pouch of Douglas were seen. The pouch of Douglas was normal. Hysteroscopy confirmed the existence of one cervix, a sort of common uterine isthmus and two separate uterine cavities (horns). Both ostia were seen and the endometrium was of normal appearance. Knowing that the paracervical pyocolpos was apparently independent, incision, drainage and marsupialisation were performed by vaginal access. A biopsy of the pseudo-cavity was taken. Incision and drainage on the anterior part of the vaginal wall had successfully treated the second purulent collection. The pathology report revealed normal malpighian epithelium and excluded malignancy and suspicion of Wolffian remnants. The patient recovered without complication in 2 days. Figure 4 illustrates the exact nature of her uterine abnormality. Her pains disappeared immediately during the first post-operative day. Broad-spectrum antibiotics (co-amoxiclav) were prescribed for 5 days and further gynaecological consultation about fertility issues was organised.

\section{Discussion}

\section{Background}

Müllerian uterine malformations although rare can surprise the gynaecologist. A bicornuate unicollis uterus after the arcuate uterus is the most common type of congenital uterine anomaly and occurs due to non-coordinated development of one of the müllerian ducts which arrests and fails to fuse with its equivalent on the other side during embryogenesis. The exact incidence of bicornuate uterus is unknown and is difficult to determine since many women with such anomalies are not diagnosed, especially if they are asymptomatic or not pregnant. The overall incidence of uterine malformations is estimated to be between 0.5 and $4 \%$ [1]. Bicornuate uteri may represent up to $25 \%$ of the müllerian malformations [1]. However, this type of uterine malformation is often diagnosed in the context of a miscarriage in the second trimester, dysmenorrhoea or delay of menarche in young girls. Existence of a vaginal septum is important to diagnose as it is often associated with ipsilateral agenesis of the kidney. We knew that her left kidney was absent and the bulgy round mass was located at the left side. The septum can give a blind hemivagina and when associated with a cervix, then a haematocolpos or pyocolpos may be formed. This is more often the case with a uterus didelphys when one of the cervixes is evacuated in a blind hemivagina. In our case no vaginal septum was localised nor a second cervix. The diagnosis of isolated pyocolpos with bicornuate uterus was made only during the minimal access surgery assessment. Periurethral or paravesical locations of an abscess have

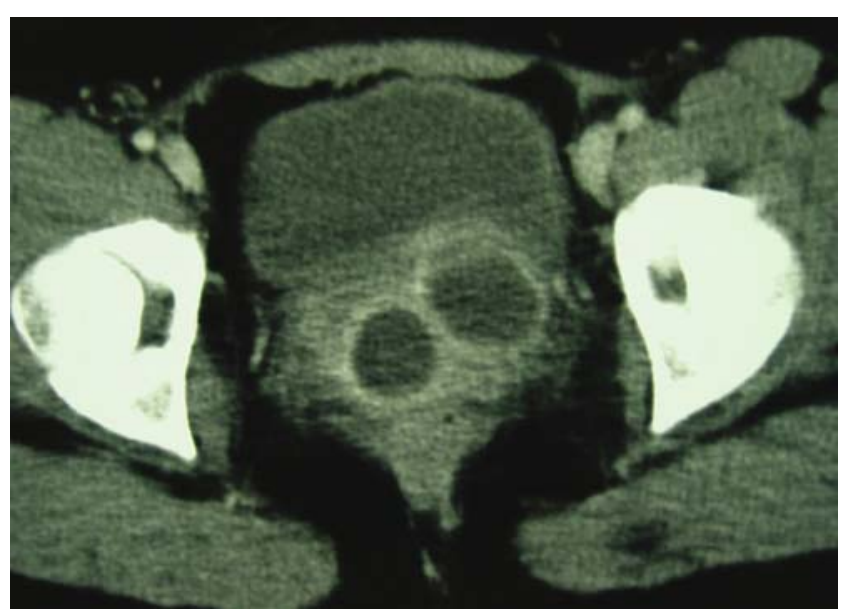

Fig. 2 CT scan images: two non-communicating paracervical abscesses 


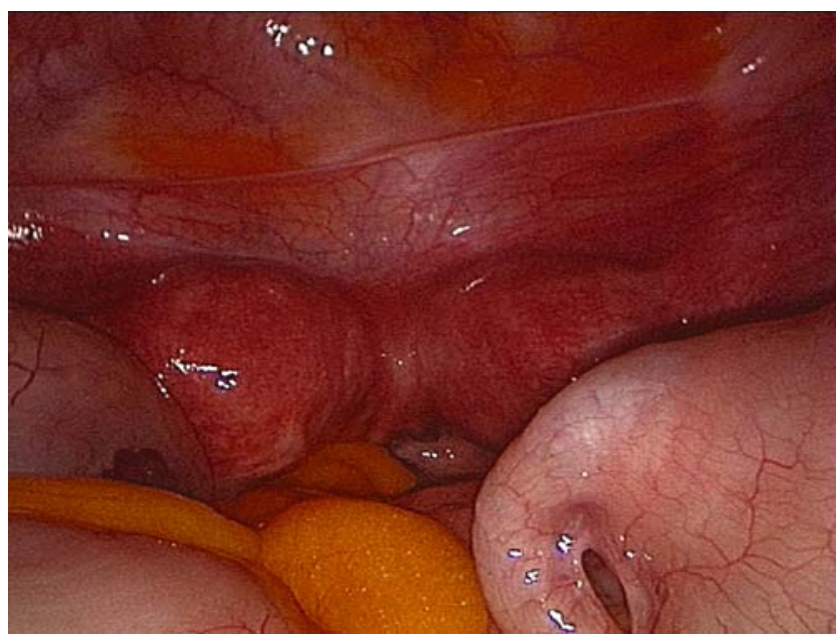

Fig. 3 Laparoscopic view: two horns and low common uterine body

been sparingly described in association with a blind vagina [2]. Uteri didelphys are associated with blind hemivaginas and haematocolpos $[3,4]$.

\section{Diagnosis}

The diagnosis of a malformation should be systematic and complete. Different classifications exist to describe the uterine malformations [5]. Most often the American Fertility Society (AFS) classification is used and in Europe most commonly Musset's classification [1]. Bicornuate uteri correspond to the class IV of the AFS classification. In order to diagnose accurately the müllerian malformation and its variations different imaging techniques should be applied. Bicornuate unicollis uterus can be seen by ultrasound scan (USS). It is better to perform the USS examination during the second part of the cycle as the endometrial thickness is higher and facilitates the diagnosis. The USS examination

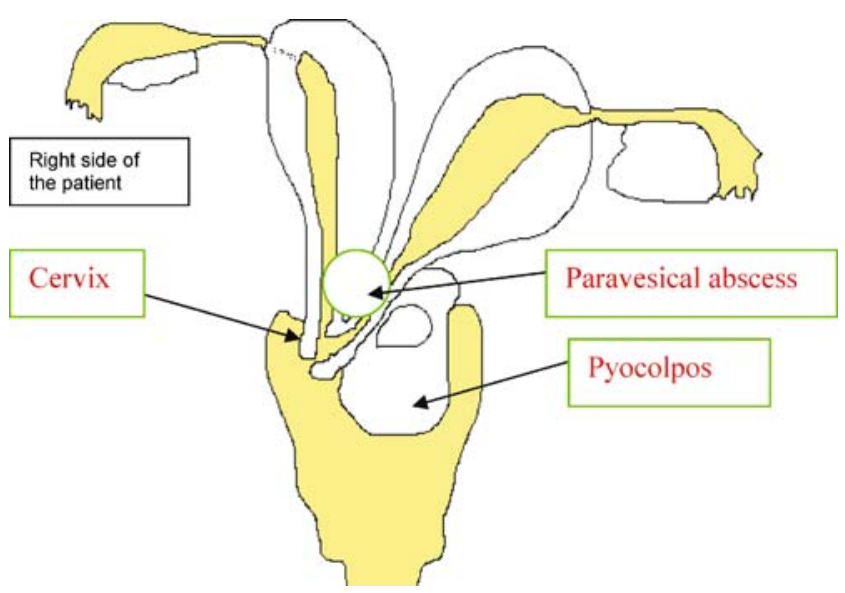

Fig. 4 Overall schematic representation: cervix deviated to the right fornix; left pyocolpos and paravesical abscess cannot reliably differentiate between bicornuate, septated or unicornuate uteri. Didelphys can be more easily seen [6]. Fibroids or other masses may sometimes be confused with müllerian anomalies. A three-dimensional ultrasound scan can contribute to the diagnosis when available [1, 7]. Magnetic resonance imaging (MRI) is the most useful complementary investigation in order to distinguish between different uterine structural malformations [8]. Careful appreciation of the endometrium versus myometrium sign and depth can help to differentiate between uterus didelphys, bicornuate uterus or septated uterus [9]. For bicornuate uterus, MRI depicts two divergent uterine horns with an increased intercornual distance of more than $4 \mathrm{~cm}$ which are separated by myometrial tissue seen on $\mathrm{T} 2$-weighted images by its intermediate signal intensity [10]. MRI and USS allow simultaneous assessment of a urinary tract anomaly. Hysterosalpingography (HSG) can contribute to the diagnosis, giving an indirect image of the interior of the uterine cavity. The differentiation between bicornuate and septated uterus is difficult some times and further imaging techniques are necessary. Suspicion of infection is a contraindication for HSG (as in our case). The characteristics of bicornuate uterus are: a fundal cleft of more than $1 \mathrm{~cm}$, a separation of uterine horns with wide intercornual angle of more than $60^{\circ}$ and more than $4 \mathrm{~cm}$ distance between maximum lateral extent of the endometrium on USS and HSG. While MRI and USS are superior to demonstrate the anatomy of the cervix, uterine body and vagina, HSG is valuable in evaluating the fallopian tube patency and intrauterine adhesions or septa [6]. However, the definitive diagnosis is done by laparoscopy and hysteroscopy. Hysteroscopy can detect the existence of an intrauterine septum and assess the uterine cavity, endometrium and ostia. In our case it was essential part for the diagnosis of bicornuate uterus as we detected the common sort of isthmus and we visualised the separated horns. Laparoscopy is essential to directly assess the external shape of the uterus and adnexa, associated endometriosis can be excluded and a dye test may be performed in order to confirm fallopian tube patency. In our case a CT scan was requested in the context of an emergency as the MRI scan could only be available with a delay of 7 days.

\section{Management}

Clear explanation and description of the malformation is essential for the patient to understand the therapeutic approach and risks. Therefore, appropriate counselling and communication skills are necessary. Drawings and images are of great value during consultation with the patient. Issues such as conservative versus radical treatment, surgical risks and difficulties or associated endometriosis must be discussed prior to any intervention. However, the approach depends on the availability and experience of the surgeon. Referral to a 
centre of excellence is advised. In our case conservative surgical management of the pyocolpos was considered at first instance taking care not to perforate the blind hemivagina. An incision evacuation is not the best approach because of the high risk of recurrence. Most of the published articles are series of cases reports and few studied the long-term prognosis of surgical treatment. Marsupialisation is successful and no recurrence has been reported in the literature. Interestingly the epithelium of the hemivagina (former pyocolpos) may undergo a non-uniform squamous metaplasia in subsequent years. Concerning pregnancy prognosis for the bicornuate unicollis uterus, the spontaneous pregnancy rates are similar to the normal uterus. However, obstetrical complications associated with bicornuate uterus are more common such as abnormal fetal lie, premature labour and growth restriction and increased miscarriage rate (first and second trimester).

\section{Conclusion}

Bicornuate unicollis uterus is one of the most common müllerian malformations. However, few cases have been reported with the primary symptom of a paracervical pyocolpos. Preoperative imaging techniques including ultrasound and MRI are necessary for the assessment of the anatomical malformation. Laparoscopic and hysteroscopic approaches should be considered at first instance. Efforts at conservative treatment and less traumatic surgery with evacuation and marsupialisation should be undertaken.

\section{References}

1. Porcu G, Heckenroth H (2005) Uterine abnormalities and infertility. EMC Gynecol Obstet 2:185-197

2. Ceccaldi PF, Ducarme G, Dedecker F, Harika G, Gabriel R, Quereux C, Graesslin O (2006) Hematocolpos due to obstructed hemivagina. About three cases (in French). Gynecol Obstet Fertil 34:510-513

3. Nisolle M, Donnez J (2001) Müllerian duct anomalies. An atlas of operative laparoscopy and hysteroscopy, 2nd edn. Operative hysteroscopy: section II, Chap. 40. Parthenon Publishing Group, Carnforth, UK, pp 409-418

4. Loumaye F, Cheruy C, Christinelli S, Foidart JM, Nisolle M (2007) Comment je traite un utérus didelphe associé à un hémivagin borgne. Rev Med Liege (in press)

5. Woodward PJ, Sohay R, Wagner BJ (1995) Congenital malformations. Curr Probl Diagn Radiol 24:178-197

6. Pui MH (2004) Imaging diagnosis of congenital uterine malformation. Comput Med Imag Grap 28:425-433

7. Salim R, Woelfer B, Backos M, Regan L, Jurkovic D (2003) Reproducibility of three-dimensional ultrasound diagnosis of congenital uterine anomalies. Ultrasound Obstet Gynecol 21 (6):578-582

8. Pellerito JS, McCarthy SM, Doyle MB et al (1992) Diagnosis of uterine anomalies: relative accuracy of MR imaging, endovaginal sonography and hysterosalpingography. Radiology 183:795

9. Imai A, Takagi H, Matsunami K (2004) Double uterus associated with renal aplasia; magnetic resonance appearance and threedimensional computed tomographic urogram. Int $\mathrm{J}$ Gynaecol Obstet 87:169-171

10. Marten K, Vosshenrich R, Funke M, Obenauer S, Baum F, Grabbe E (2003) MRI in the evaluation of müllerian duct anomalies. Clin Imaging 27:346-350

11. Candiani GB, Fedele L, Candiani M (1997) Double uterus, blind hemivagina and ipsilateral renal agenesis: 36 cases and long-term follow-up. Obstet Gynecol 90(1):26-32 